\title{
Studying Cosmic Ray Composition with IceTop using Muon and Electromagnetic Lateral Distributions
}

\author{
The IceCube Collaboration ${ }^{\dagger}$ \\ ${ }^{\dagger}$ http://icecube.wisc.edu/collaboration/authors/icrc15_icecube \\ E-mail: jgonzalezaicecube.wisc.edu
}

\begin{abstract}
In this contribution we will consider the methods at our disposal to estimate the mass of primary cosmic rays on an event-by-event basis using IceTop, the surface component of the IceCube detector at the geographical South Pole. Events are reconstructed using two lateral distribution functions, one for the muon component and one for the electrons and gamma rays. This results in a few parameters that are sensitive to primary mass: the muon density at large lateral distances and the steepness of the lateral distribution of the electromagnetic component of the air shower. This approach is complementary to the technique already used in IceCube, whereby one can get a mass-sensitive parameter using the air shower size in IceTop together with several observables from the deep portion of the detector. Most importantly, this approach allows the study of composition-dependent anisotropy, since the zenith angle range is not constrained by the requirement of detecting the air shower in the deep detector.
\end{abstract}

Corresponding author: J. G. Gonzalez

Bartol Research Institute, University of Delaware, USA

The 34th International Cosmic Ray Conference,

30 July- 6 August, 2015

The Hague, The Netherlands 
It is well known that the muon content of an air shower, together with a measure of its electromagnetic component, can be used to estimate the energy and mass of its primary [1]. The main issue with the use of the muon content as an estimate of primary mass is the possible systematic differences between simulated and real air showers, arising from the lack of knowledge of high energy hadronic interactions. The IceTop detector is sensitive to the low-energy ( $\mathrm{E} \gtrsim 200 \mathrm{MeV}$ ) muon component of air showers [2]. Generally speaking, for near-vertical air showers and close enough to their axis, the signal from muons is overwhelmed by the signal from the electromagnetic (EM) component of the air shower (electrons, positrons and gamma-rays). This holds true in the zenith angle and lateral distance ranges that have been used in the cosmic ray spectrum determination with IceTop $[3,4,5]$, where the lateral distance of any point is defined as the closest distance from the point to the shower axis.

In this article, signals recorded at large lateral distances are included for the first time in the event-by-event analysis of air showers recorded with IceTop. These signals are collected using a dedicated readout mode introduced in 2010 (Section 1). The general features of IceTop and the signals at large lateral distances are briefly described in Section 1 and their sensitivity to the muon content of the air shower is briefly reviewed in Section 2. This new analysis thus provides an extra observable which correlates to the number of muons in the air shower.

\section{General Features of IceTop}

The IceCube detector consists of two major components [6, 7]. It can measure air showers on the surface with IceTop, high energy muon bundles with the in-ice detector, and both components in coincidence provided that the air shower axis goes through both the surface and in-ice detectors. The specific characteristics of IceTop that are relevant for measuring the low-energy muon component of air showers is described in what follows. A more detailed description of IceCube and IceTop has already been presented elsewhere [7].

IceTop is an air shower array consisting of 81 stations forming a triangular grid with a separation of $125 \mathrm{~m}$ in its completed configuration. The results presented here were obtained with data collected between June 1st 2010 and May 13th 2011, when IceTop consisted of 73 stations. It is located above the deep IceCube detector at the geographical South Pole, covering an area of roughly one square kilometer. Each station consists of two ice Cherenkov tanks separated by ten meters. Each tank contains two Digital Optical Modules (DOMs) with a 10 inch photomultiplier tube (PMT) and electronics for signal processing and readout. A discriminator trigger occurs when the voltage in one of the DOMs in a tank has passed the discriminator threshold. The total charge collected at the PMT's anode, after digitization and baseline subtraction, constitutes the tank's signal. The tanks register signals ranging from 0.2 to 1000 Vertical Equivalent Muons (VEM). A Hard Local Coincidence (HLC) occurs when there are discriminator triggers in two neighboring tanks within a time window of $1 \mu \mathrm{s}$. If there is a discriminator trigger but not an HLC, the result is a Soft Local Coincidence (SLC).

In previously-published analyses, including those measuring the all-particle spectrum and other IceTop analyses $[3,4,5]$, the properties of the primary cosmic ray are reconstructed by first fitting the signal times with a function describing the shape of the shower front and then fitting the measured signals with a single Lateral Distribution Function (LDF) which includes an attenuation 


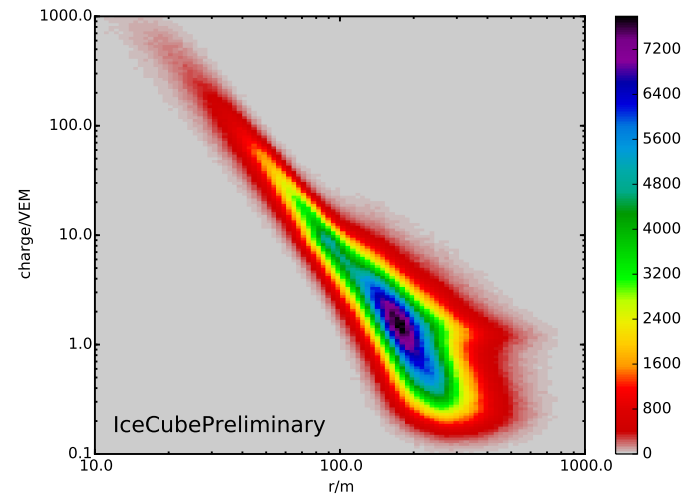

(a) Signal distribution as a function of lateral distance for air showers with energies between 4 and $5 \mathrm{PeV}$, and zenith angle between $28^{\circ}$ and $32^{\circ}$. This distribution is a $2 \mathrm{~d}$ histogram that includes only HLC signals from all events with the given energy and arrival direction.

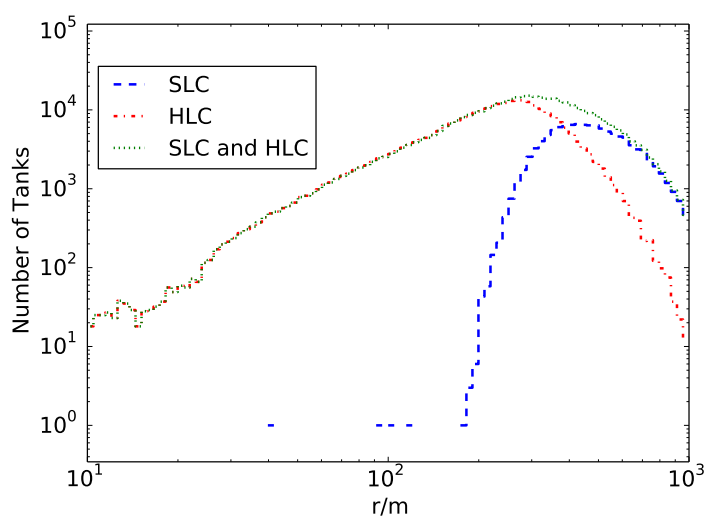

(b) Lateral distribution of signals in showers arriving with zenith angles less than $6^{\circ}$ and with energies between $10 \mathrm{PeV}$ and $12.6 \mathrm{PeV}$. The signals are classified depending on whether they are $S L C$ or $H L C$ signals (described in the text).

Figure 1: Lateral distribution of signals in IceTop

factor due to the snow cover on top of each tank [8]. The primary energy is given by the shower size $S_{125}$, defined as the signal interpolated at a lateral distance of $125 \mathrm{~m}$. Only HLC signals were considered in these analyses. An example of the observed lateral distribution of HLC signals in IceTop data after selection-fiducial cuts on core location and on signal, and signals selected in a time window around the expected shower front time-is shown in Figure 1a for air showers with energies between 4 and $5 \mathrm{PeV}$, and zenith angle between $28^{\circ}$ and $32^{\circ}$.

The main improvement described in this contribution is the addition of SLC signals, those where the partner tank within the station did not have a discriminator trigger. SLC signals occur at large lateral distances, where the triggering probability is smaller. An example of the lateral distribution of SLC and HLC signals from experimental data is shown in Figure 1b. The distinction between SLCs and HLCs provides a natural way to identify tanks where one expects to see a larger muon contribution to the signal. Generally speaking, one expects that signals at large lateral distances will be mostly due to muons, whereas the signals at short lateral distances will be mostly due to electrons and gamma-rays.

The sensitivity of SLC to muons can be seen in Figure 2, where the lateral distribution of SLC and all signals in experimental data is displayed. Note especially how the SLC signals follow a bimodal distribution. They can be described as two populations of signals. One population is the continuation of the main distribution at smaller distances, which roughly follows a power law, where the electromagnetic component of the shower dominates. The other population, with signals around 1 VEM, is made up mostly of tanks hit by one or more muons.

\section{Modelling the Signal Distribution at Large Lateral Distances}

In order to use SLC signals in the reconstruction procedure, the statistical signal distribution 


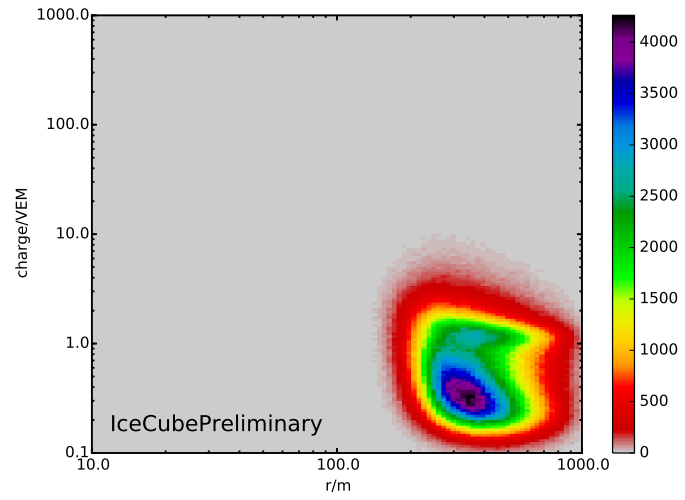

(a) SLC signals only.

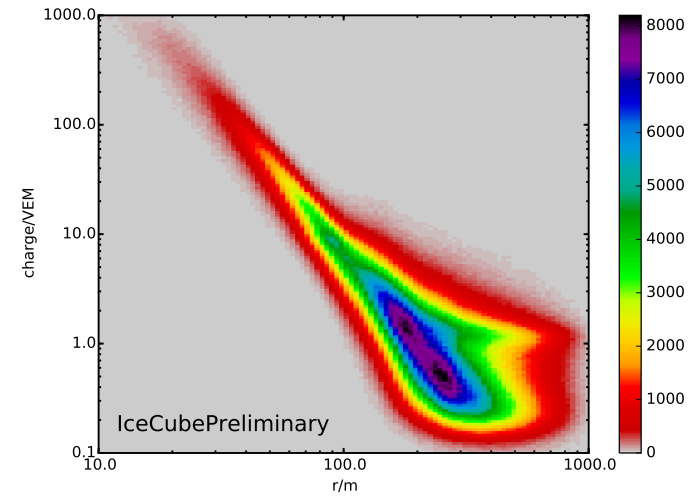

(b) SLC and HLC signals.

Figure 2: Signal distribution as a function of lateral distance for air showers with energies between 4 and $5 \mathrm{PeV}$, and zenith angle between $28^{\circ}$ and $32^{\circ}$. Same as previous figure but including SLCs.

for low expected signals needs to be understood. This distribution is already used to estimate the average lateral distribution of muons at fixed energies and zenith angles [2,9]. It is used now to produce a probability model of the signals in single events. This model is then used to implement a likelihood-based reconstruction procedure that yields a muon number for each event (Section 3).

The characteristic features of the signal distribution at large lateral distances from the shower axis are mostly determined by the muon LDF. The muon LDF in IceTop can be described by the following function [10]:

$$
\rho_{\mu}(r)=\rho_{\mu}\left(r_{0}\right)\left(\frac{r}{r_{0}}\right)^{-3 / 4}\left(\frac{320 \mathrm{~m}+r}{320 \mathrm{~m}+r_{0}}\right)^{-\gamma},
$$

which displays the same functional form as Greisen's function, with the first exponent of $r$ fixed to $-3 / 4$, and $r_{0}$ set to $600 \mathrm{~m}$. It must be noted that the optimum value for $r_{0}$ depends on energy. The $\gamma$ parameter potentially depends on energy and zenith angle but one can use Greisen's value of 2.5. For a more detailed description, refer to the dedicated contribution in these proceedings [9].

The distribution of signals from EM particles (electrons and gamma rays) will roughly mimic their energy distribution, with a mean signal that corresponds to a few tens of centimeters of track length inside the tank. On the other hand, the distribution of signals from muons is mainly determined by the geometry of the tank. The signal distributions produced by single muons are obtained using the Geant4 toolkit [11]. Example distributions at various incident angles are displayed in Figure 3a. The distributions are clearly not symmetric. The peak of the distribution corresponds to muons that enter through the top of the tank and exit through the bottom. By definition, the peak position for vertically through-going muons is one Vertical Equivalent Muon (VEM). For muons arriving at a zenith angle $\theta$, the peak is at $1 / \cos (\theta)$. The flat part at low signal values in Figure $3 \mathrm{a}$ corresponds to muons with a short track through the tank, known as corner clipping muons. At large angles, few muons go through top and bottom. For an integer number of muons, the signal distribution is just the multiple auto-convolution of the single-particle distribution. An example of this is displayed in Figure $3 b$. The statistical distribution of the total signal $\mathrm{S}$ for an expected aver- 


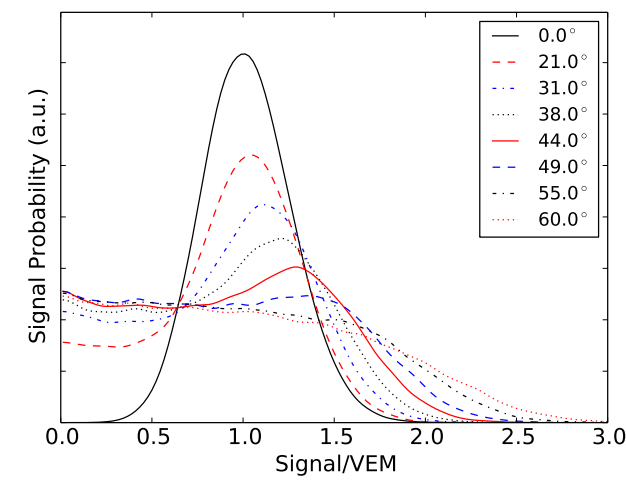

(a) Signal distributions resulting from simulating the detector response for single muons arriving at fixed zenith angles from $0^{\circ}$ to $57^{\circ}$.

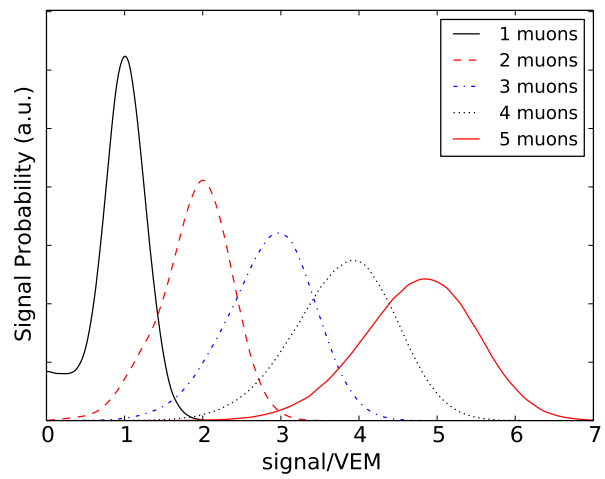

(b) Signal distributions resulting from simulating the detector response for an integer number of muons arriving arriving at $10^{\circ}$.

Figure 3: Detector response to muons.

age number of muons $\left\langle N_{\mu}\right\rangle$ is given by a linear combination of the signal distributions for integer numbers of muons:

$$
p_{\text {pois }}\left(S \mid\left\langle N_{\mu}\right\rangle\right)=\sum_{n=0}^{\infty} \frac{\left\langle N_{\mu}\right\rangle^{n}}{n !} e^{-\left\langle N_{\mu}\right\rangle} p\left(S \mid N_{\mu}=n\right)
$$

\subsection{The Likelihood Function}

The concepts just described are included in a likelihood function of the air shower size, electromagnetic shower age, muon number, arrival direction, and core location. The likelihood function in this $t w o-L D F$ model of air showers takes the form:

$$
\mathscr{L}\left(S_{r_{0}}, s, \rho_{r_{0}}, \theta, \phi, \vec{r}_{c}\right)=\prod_{i} p\left(S_{i} \mid \theta,\left\langle S_{e m}\left(\vec{r}_{i}\right)\right\rangle,\left\langle S_{\mu}\left(\vec{r}_{i}\right)\right\rangle\right),
$$

where the index $i$ labels all tanks in the array, with or without signal. $S_{e m}(\vec{r})$ and $S_{\mu}(\vec{r})$ are lateral distribution functions of the electromagnetic and muon components respectively. They are given by NKG-like functions

$$
\begin{aligned}
\left\langle S_{\mu}\right\rangle & =\rho_{\mu}(r)=\rho_{r_{0}}\left(\frac{r}{r_{0}}\right)^{-0.75}\left(\frac{r+r_{m \mu}}{r_{0}+r_{m \mu}}\right)^{-\gamma} \\
\left\langle S_{e m}\right\rangle & =S_{r_{0}}\left(\frac{r}{r_{0}}\right)^{s-2}\left(\frac{r+r_{m}}{r_{0}+r_{m}}\right)^{s-4.5}
\end{aligned}
$$

in which the relevant parameters are the shower age $s$, the electromagnetic signal at a reference radius $S_{r_{0}}$ and the muon density at a refernce radius $\rho_{r_{0}}$. The $r_{0}$ parameter is an arbitrary constant that is absorbed in the normalization of the function. The Moliere radius $r_{m}$ is a constant that depends on the arrival direction and the air density profile. It can be determined for each event. The equivalent number in the muon LDF $r_{m \mu}$ is an empirical number that is fixed to $320 \mathrm{~m}$, the 
value determined by Greisen. The parameter $\gamma$ is fixed to 2.5 , the value from Greisen. Note that the expected muon signal in VEM is just the muon density. The number of muons going through the tank is $N_{\mu}=\rho_{\mu}(r)\left(A_{\text {top }} \cos \theta+A_{\text {side }} \sin \theta\right)$.

The probability of getting an electromagnetic signal, when $\left\langle S_{e m}\right\rangle$ is expected, is given by a normal distribution centered at $\left\langle S_{e m}\right\rangle$ with a width $\sigma_{e m}=0.3\left\langle S_{e m}\right\rangle^{1 / 2}$ and the probability of measuring a muon signal is given the muon density as described in Section 1. In other words:

$$
\begin{aligned}
p_{\mu}\left(S \mid \theta,\left\langle S_{\mu}\right\rangle\right) & =\sum_{n=1}^{\infty} p_{\text {det }}(S \mid \theta, n) \cdot p_{\text {pois }}\left(n \mid\left\langle N_{\mu}\right\rangle\right) \\
p_{e m}\left(S \mid \theta,\left\langle S_{e m}\right\rangle\right) & =\frac{1}{\sigma_{e m} \sqrt{2 \pi}} e^{-\left(S_{e m}-\left\langle S_{e m}\right\rangle\right)^{2} / 2 \sigma_{e m}^{2}}
\end{aligned}
$$

where $p_{\text {det }}(S \mid \theta, n)$ is a tabulated response function like the one depicted in Figure $3 \mathrm{~b}$ and $p_{\text {pois }}\left(n \mid\left\langle N_{\mu}\right\rangle\right)$ is given by Eq.2.2. Using these functions, the probability of recording a signal $S$ at a given lateral distance and arrival direction is

$$
p\left(S \mid \theta,\left\langle S_{e m}\right\rangle,\left\langle S_{\mu}\right\rangle\right)=p_{t r}(S) \cdot \int_{0}^{s} p_{e m}\left(S_{e m} \mid \theta,\left\langle S_{e m}\right\rangle\right) \cdot p_{\mu}\left(S-S_{e m} \mid \theta,\left\langle S_{\mu}\right\rangle\right) d S_{e m},
$$

where $p_{t r}(S)$ represents the discriminator trigger probability, which is parametrized as

$$
p_{t r}(S)=\frac{1}{2}\left(\operatorname{erf}\left(\frac{\log (S)+0.66}{0.14 \sqrt{2}}\right)+1\right)
$$

\subsection{The Effect of Snow}

The effect of the snow on the detection process is accounted for by multiplying the expected signal by an attenuation factor parametrized from Monte Carlo simulations. This factor depends on snow cover, primary energy, arrival direction, lateral distance and the distance from the detector to shower maximum. It is described in a separate contribution [8]. The muon component is assumed not to be affected by the presence of snow, and only the effect on the electromagnetic component is considered. If the expected EM signal is $S_{e m}$, the expected EM signal after going through the snow $S_{e m}^{\prime}$ is

$$
S_{e m}^{\prime}=c_{\text {snow }} S_{e m}
$$

and 2.8 becomes

$$
p\left(S \mid \theta,\left\langle S_{e m}\right\rangle,\left\langle S_{\mu}\right\rangle\right)=\int_{0}^{s} p_{e m}\left(S_{e m}^{\prime} / c_{\text {snow }} \mid \theta,\left\langle S_{e m}\right\rangle\right) \cdot p_{\mu}\left(S-S_{e m}^{\prime} \mid \theta,\left\langle S_{\mu}\right\rangle\right) d S_{e m}^{\prime} .
$$

The attenuation due to snow is parametrized in terms of shower evolution stage $t$, the distance between the observation point and the shower maximum in units of radiation length in air $\left(X_{0}=\right.$ $36.7 \mathrm{~g} / \mathrm{cm}^{2}$ ). It is possible, within this model of air showers, to relate the stage parameter to the shower age. If one assumes a Greisen profile, they are related by:

$$
t=\frac{1}{2} \frac{X}{X_{0}}\left(1+\frac{3}{s}\right)
$$

where $X$ represents the slant depth of the observation point. The effect of this correction is under study. 


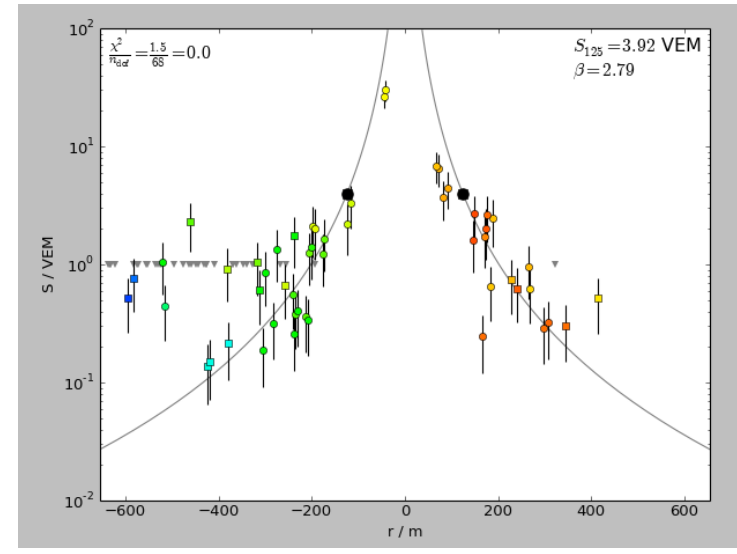

(a) Using the single-LDF reconstruction

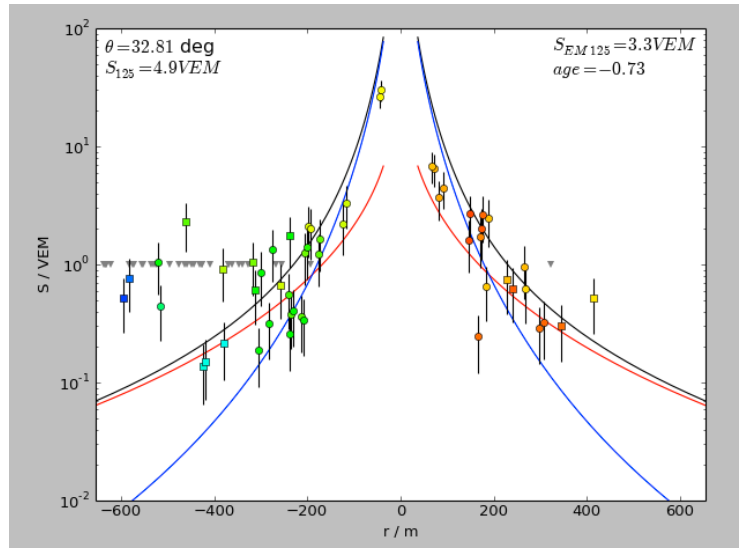

(b) Using two LDFs as described in text

Figure 4: Reconstructed lateral distribution using the two methods: the single-LDF (a) and two$L D F(b)$. Note that the signals depicted as squares are SLCs and the circles are HLCs. SLCs are not used in the single-LDF reconstruction. The triangles mark the locations of tanks that did not register a signal even though they were functioning. The blue line in (b) denotes the electromagnetic LDF while the red line denotes the muon LDF. The black line is the sum of the EM and muon LDF.

\section{Event by Event Reconstruction and Results}

The reconstruction of IceTop events proceeds by first selecting the events according to standard selection criteria, and reconstructing them with a single-LDF using only HLC signals [3]. Standard selection criteria consist of fiducial cuts on core location and on signal. This gives the initial values for the core location, arrival direction and shower size. At this point, SLC signals are selected based on their agreement in time with the arrival of the air shower. This removes most random background coincidences. Finally, a two-LDF model is fit to the signals in the event, maximizing the log-likelihood function described in section 2.1.

A typical reconstructed event is shown in Figure 4. For comparison, the same event is shown with the single-LDF and the two-LDF reconstructions. As already noted, the single LDF reconstruction does not consider SLC signals (marked as squares in Figure 4). In this figure, the two branches of the LDF denote the early and late parts of the air shower. The filled gray triangles denote the location of tanks that had no signal. This particular event has most tanks-with or without signal - in the late part of the shower due to purely geometric reasons. Note the signals at large lateral distances around 1 VEM. Figure $4 \mathrm{~b}$ shows three lines, the blue line represents the EM component, the red line represents the muon component, and the black line is the sum of the EM and muon LDFs. As expected, at short lateral distances the electromagnetic LDF dominates and determines the values of the HLC signals (circles), while at large lateral signals the muon LDF dominates, determining the probability that a tank has a signal and the values of the SLC signals (squares). Note also how the blue line follows the position of the small signals between 200 and $400 \mathrm{~m}$ from the core. It is important to keep in mind that the red line is not expected to go right through the points betwen 500 and $600 \mathrm{~m}$. The red line gives the expected number of muons, which are either present—giving signals around 1 VEM—or there is no signal at all. For the same reason, 
the black line does not follow the points. It includes information on tanks without signal.

\section{Outlook}

The method described here uses SLC signals detected in IceTop in the analysis of individual air shower events. The inclusion of this kind of signals in the reconstruction has the potential to yield independent observables that are sensitive to the mass of the primary. The obvious one is the muon number at a fixed radius $\rho_{r_{0}}$, but the use of an NKG function for the EM component provides an age parameter. The sensitivity to composition arising from these two parameters is still under investigation.

The signal model relies on splitting the air shower in two components, a muon component and an electromagnetic component, but one can foresee improvements on this approach by considering multiple components in a way similar to the one described by Ave et al. [12].

\section{References}

[1] K.-H. Kampert and M. Unger, Astropart.Phys. 35 (2012) 660-678, [arXiv: 1201.0018 ].

[2] IceCube Collaboration, J. G. Gonzalez et al., Measuring the Muon Content of Air Showers with IceTop, in Proc. of the 18th meeting of the International Symposium on Very High Energy Cosmic Ray Interactions (ISVHECRI 2014), 2014. arXiv:1501.0341.

[3] IceCube Collaboration, R. Abbasi et al., Astropart.Phys. 44 (2013) 40-58, [arXiv: 1202.3039 ].

[4] IceCube Collaboration, B. Ruzybayev et al., Cosmic ray composition and energy spectrum between $2.5 \mathrm{PeV}$ and $1 \mathrm{EeV}$, in Proc. of the 33rd ICRC \#246, Rio de Janeiro, Brasil, 2013. arXiv:1309.7006.

[5] IceCube Collaboration, T Feusels et al., Measurement of the cosmic ray energy spectrum with IceTop 73, in Proc. of the 33rd ICRC \#861, Rio de Janeiro, Brasil, 2013. arXiv:1309. 7006.

[6] IceCube Collaboration, A. Achterberg et al., Astropart.Phys. 26 (2006) 155-173, [astro-ph/0604450].

[7] IceCube Collaboration, R. Abbasi et al., Nucl.Instrum.Meth. A700 (2013) 188-220, [arXiv:1207.6326].

[8] IceCube Collaboration, K. Rawlins et al., A Function to Describe Attenuation of Cosmic Ray Air Shower Particles in Snow, PoS(ICRC2015)628 these proceedings.

[9] IceCube Collaboration, H. Dembinski and J. Gonzalez et al., Surface Muons in IceTop, PoS(ICRC2015)267 in these proceedings.

[10] K. Greisen, Annual Review of Nuclear Science 10 (1960) 63.

[11] S. Agostinelli et al., Nuclear Instruments and Methods in Physics Research A 506 (2003) 250-303.

[12] M. Ave et al., Extensive air shower universality of ground particle distributions, in Proc. of the 32nd ICRC, Beijing, China, 2011. 